\title{
NONLINEAR DOUBLE DIFFUSIVE CONVECTION FROM A RADIATIVE SLENDER PARABOLOID IN A NON-DARCY POROUS MEDIUM
}

\author{
RISHI RAJ KAIRI ${ }^{1}$, CH. RAMREDDY ${ }^{2 *}$ \\ ${ }^{1}$ Mathematics Department, Cooch Behar Panchanan Barma University, \\ Cooch Behar-736101, India \\ ${ }^{2}$ Mathematics Department, National Institute of Technology \\ Warangal-506004, India
}

[Received: 09 July 2019. Accepted: 30 April 2020]

doi: 10.7546/JTAM.50.20.03.01

\begin{abstract}
This paper describes the nonlinear double diffusive convective phenomena of a Ostwald-de Waele power-law fluid from a radiative slender paraboloid in a non-Darcy porous medium. A suitable set of similarity transformations is utilized to convert the set of nonlinear partial differential equations to the set of nonlinear ordinary differential equations and hence, the resultant equations are solved numerically. The significance of various flow influencing parameters on dimensionless velocity, concentration, temperature, and heat and mass transfer rates, is elaborated graphically in detail.
\end{abstract}

KEY WORDS: Radiation, Nonlinear Convection, Power-Law Fluid, Non-Darcy Porous Medium, Slender Body.

\section{INTRODUCTION}

In nature, the existence of non-uniform or arbitrary shaped bodies is many times more in number than the uniform shaped bodies. Due to the arbitrariness in the shape of bodies, it is quite difficult task to find mathematical model in a particular situation. As a result, an extensive research in the field of convective transport occurring from uniform shaped bodies has been reported by researchers. Furthermore, the transport process of heat and mass due to convection becomes complicated when it is supposed to occur from arbitrary or axisymmetric bodies and when it is compared with that of a definite shaped bodies.

Though a numerous research has been reported in the study of convective transport phenomena of non-Newtonian fluids but due to huge arena of applicability in industries, the researchers are still involved themselves to investigate transport mechanism of energy and solute for such fluids. Many researchers [1-4] examined transport process of non-Newtonian fluid over different solid geometries in Darcy or non-Darcy

\footnotetext{
${ }^{*}$ Corresponding author e-mail: chittetiram@gmail.com; chramreddy@nitw.ac.in
} 
porous medium. Nonlinear relation between the shear stress and rate of shear of nonNewtonian fluid is characterized by Ostwald-de Waele power-law fluid model by several investigators [5-7] while studying convective transport of non-Newtonian fluid in porous medium. Energy and solutal diffusion of non-Newtonian fluids through porous medium finds enormous application in geophysical and thermal engineering related problems. In this direction, a similarity solution of unsteady flow over axisymmetric bodies of non-Newtonian fluids was presented by Mohanty [3]. Nakayama and Koyma [5] reported that any two dimensional/axisymmetric body offers similarity solution while surface temperature follows a particular class of distribution. Later on Nakayama and Koyama [8] further extended their work to investigate the free convective transport of non-Newtonian fluids. Double diffusive convective transport of heat and mass from slender paraboloid and cylinder was elaborately discussed by Lai et al. [9]. While, Singh and Chandrika [10] used integral method of Von-Karman type to obtain the solution of the same problem which was reported by Lai et al. [9]. It is noticed that the integral treatment also justified the results of Lai et. al. [9] which was obtained numerically by Runge-Kutta method. Considering some geothermal and oil reservoir engineering applications in porous medium, the analysis of temperature distribution of power-law fluids without and/or with yield stress was nicely elaborated by Shenoy $[8,11]$. Recently, Babu and Sandeep [4] examined cross-diffusion effects on a slendering stretching sheet in a power-law fluid in the presence of magnetohydrodynamics. Reddy et al. [12] showed the consequences of frictional heating on the flow of a radiative ferrofluid over a slendering stretching body. In this direction, Reddy et al. [13] investigated role of convective heat transport of a Casson fluid flow over a heated paraboloid of revolution.

Radiative heat transfer from heated bodies caused by surface characteristics and solid geometry is quite significant while investigating convective transport phenomena in a porous medium. Raptis [14] used Rosseland approximation to analyze the radiation effect on natural convection in a porous medium. Assuming power-law variation in the surface temperature, Mohammadein and El-Amin [7] reported that heat transfer rate reduces with increased values of the power-law index parameter, while the same increases with an increase in the exponent associated with the surface temperature.

At high temperature with the different second order effects namely, viscous dissipation, radiation or inertia etc., the density of the fluid varies as a nonlinear function of temperature and concentration (widely termed as nonlinear Boussinesq approximation). Considering this nonlinear approximation, several researchers [15-17] tried to analyze the heat transport phenomena in the presence of some second order effects and some useful observations are noticed. In Parta [17], the correlations of dispersion and cross-diffusion effects were analyzed numerically by considering the nonlinear 
Boussinesq approximation. Mandal and Mukhopadhyay [18] examined the role of nonlinear convection on an exponentially stretching sheet in a micropolar fluid. They concluded that the nonlinear thermal convection parameter plays an important role to enhance velocity inside the boundary layer while the same diminishes angular velocity and temperature.

Several researchers [19-22] showed their interest in analyzing the impact of chemical reaction on heat and mass transport process in a porous medium by considering flows about different flow geometry. Modather et al. [19] examined an oscillatory MHD flow of micropolar fluid past a permeable vertical plate in a porous medium. They found that the skin friction and couple stress coefficients at the surface are decreased with mounting values of chemical reaction parameter whereas the reverse effect was seen with increased values of permeability parameter. Rashad et al. [20] reported that the chemical reaction tends to diminish heat and mass transfer rates for mixed convection flow about a sphere in porous medium. Recently, Kairi [23] conducted a study on radiative heat transport around a slender paraboloid of revolution in a non-Newtonian fluid saturated porous medium. It has been stated that the local Nusselt number is reduced with a rise in the radius of slender body. Moreover, the performance of radiation becomes less important with increasing value of power-law index.

In this paper, we intend to explore the nonlinear double diffusive convective flow around a radiative slender paraboloid in a non-Darcy porous medium saturated by non-Newtonian fluids. Nonlinear Boussinesq approximation is employed to express fluid density as a nonlinear function of temperature and concentration. Boundary layer equations are transformed by introducing similarity variables. Resultant coupled system of ordinary differential equations, is solved with the help of 4th order Runge-Kutta method along with the shooting technique. Finally, the important observations of different flow controlling parameters on the flow, concentration, temperature, and the local Sherwood and Nusselt numbers are shown graphically.

\section{MATHEMATICAL FORMULATION}

Consider a two-dimensional laminar, steady flow of a non-Newtonian fluid over a slender paraboloid embedded in a homogeneous and isotropic porous medium. The power-law form is adopted to describe the nonlinear relation between the shear stress and rate of shear of non-Newtonian fluid. The saturating fluid in porous medium is supposed to be absorbing-emitting radiation and gray without scattering. Porous medium is considered to be clear and in thermal equilibrium with the saturated fluid. Temperature and concentration of the fluid near to the surface of the paraboloid are taken to be $T_{w}$ and $C_{w}$ respectively, whereas at the ambient media the temperature and concentration are taken to be $T_{\infty}$ and $C_{\infty}$ respectively. The fluid flow in the 


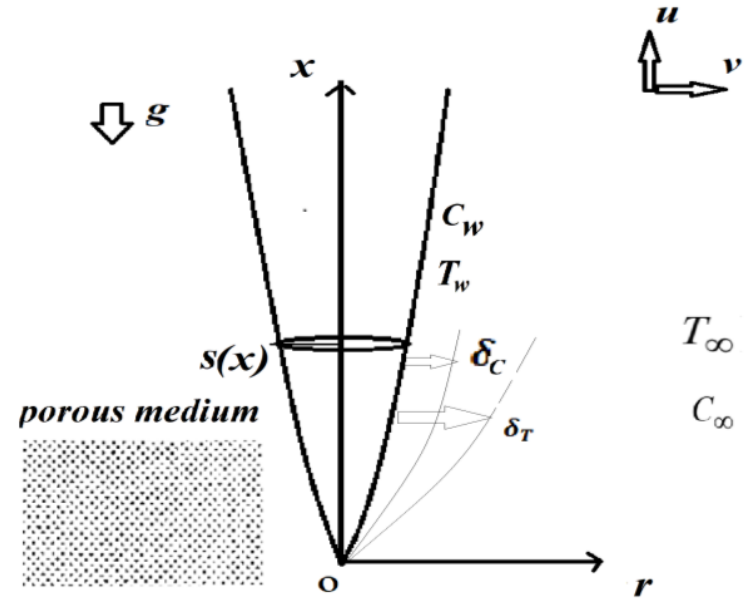

Fig. 1: Physical model and co-ordinate system.

porous medium is taken to be moderate so that the Forchheimer model is appropriate and the boundary drag is negligible.

Many researchers have considered the free or mixed convective flows over different shaped bodies in various fluid flow situations by taking the linear Boussinesq approximation, that is by taking density as a linear function of temperature and concentration. But, involvement of various second order effects like radiation, inertia, viscous dissipation etc., in different fluids saturated porous medium induces a considerable variation in density gradient. Thus researchers namely, Parth [17], Srinivasacharya et al. [15], Ch. Ramreddy et al. [16] assumed nonlinear Boussinesq approximation for the variation in density while studying convection transport in a porous medium. The nonlinear Boussinesq approximation is expressed as

$\rho=\rho_{\infty}\left(1-\beta_{0}\left(T-T_{\infty}\right)\left[1+\frac{\beta_{1}}{\beta_{0}}\left(T-T_{\infty}\right)\right]-\beta_{2}\left(C-C_{\infty}\right)\left[1+\frac{\beta_{3}}{\beta_{2}}\left(C-C_{\infty}\right)\right]\right)$.

Assuming the above approximation, the governing equations of flow, energy and concentration may be written as

$$
\frac{\partial(r u)}{\partial x}+\frac{\partial(r v)}{\partial r}=0
$$

(2) $\frac{\partial u^{n}}{\partial r}+\frac{\rho_{\infty} b K^{*}}{\mu} \frac{\partial u^{2}}{\partial r}=$

$$
=\frac{K^{*} \rho_{\infty} g}{\mu}\left[\left(\beta_{0}+2 \beta_{1}\left(T-T_{\infty}\right)\right) \frac{\partial T}{\partial r}+\left(\beta_{2}+2 \beta_{3}\left(C-C_{\infty}\right)\right) \frac{\partial C}{\partial r}\right],
$$




$$
\begin{gathered}
u \frac{\partial T}{\partial x}+v \frac{\partial T}{\partial r}=\frac{\alpha}{r} \frac{\partial}{\partial r}\left(r \frac{\partial T}{\partial r}\right)-\frac{1}{\rho c_{p}} \frac{\partial}{\partial r}\left(r q_{r}\right), \\
u \frac{\partial C}{\partial x}+v \frac{\partial C}{\partial r}=\frac{D}{r} \frac{\partial}{\partial r}\left(r \frac{\partial C}{\partial r}\right),
\end{gathered}
$$

where $S(x)=\left\{\frac{\alpha \mu^{*}}{\rho_{\infty} K^{*}} g \beta_{0}\left(T_{w}-T_{\infty}\right)\right\}\left(x \eta_{0}\right)^{1 / 2}$ represents the shape of paraboloid surface, $r$ and $x$ are the radial and axial distance co-ordinates, $v$ and $u$ are the velocity components along $r$ and $x$ directions in order, $\alpha$ and $D$ describe the effective thermal and solutal diffusivity respectively, $\rho_{\infty}$ indicates some reference density, $\beta_{0}$ and $\beta_{1}$ $\left(\beta_{2}\right.$ and $\left.\beta_{3}\right)$ denote the 1 st and 2 nd order thermal (solutal) expansion coefficients, respectively, $C$ and $T$ are the concentration and temperature respectively, $g$ is the acceleration due to gravity and $n$ is the power-law index ( $n>1, n=1$ and $n<1$ indicate dilatant, Newtonian and pseudoplastics fluids respectively). $K^{*}$ denotes the modified permeability of the porous medium which is a function of power-law index $n$, well defined in Christopher and Middleman [24] and Dharmadhikari and Kale [25].

The associated boundary conditions are

$$
\begin{array}{lll}
\text { at } & r=S(x): & v=0, T=T_{w}, C=C_{w} . \\
\text { as } & r \rightarrow \infty: & u=0, T \rightarrow T_{\infty}, C \rightarrow C_{\infty} .
\end{array}
$$

Here the suffixes $w$ and $\infty$ stand for the conditions at the surface of the body and at the ambient medium, respectively.

In order to minimize the number of variables, we define the stream function $\psi(x, r)$ in such a way that $u=\frac{1}{r} \frac{\partial \psi}{\partial r}$ and $v=-\frac{1}{r} \frac{\partial \psi}{\partial x}$.

The radiation term appearing in equation (3) can be expressed by Rosseland approximation as

$$
q_{r}=-\left(\frac{4 \sigma^{*}}{3 \kappa}\right) \frac{\partial T^{4}}{\partial r} .
$$

Introducing equation (7) into the equation (3), we get

$$
u \frac{\partial T}{\partial x}+v \frac{\partial T}{\partial r}=\frac{\alpha}{r} \frac{\partial}{\partial r}\left(r \frac{\partial T}{\partial r}\right)+\frac{4 \sigma^{*}}{3 \kappa} \frac{1}{\rho c_{p}} \frac{\partial}{\partial r}\left(r \frac{\partial T^{4}}{\partial r}\right),
$$

where $\sigma^{*}$ and $\kappa$ represent the Stefan-Boltzman and mean absorption coefficients, respectively.

Making use of Taylor series expansion of $T^{4}$ about $T_{\infty}$ and neglecting higher order terms, we get

$$
T^{4}=4 T_{\infty}^{3} T-3 T_{\infty}^{3}
$$


Using (9) in (8), we get the transformed energy equation as

$$
u \frac{\partial T}{\partial x}+v \frac{\partial T}{\partial r}=\frac{\alpha}{r} \frac{\partial}{\partial r}\left(r \frac{\partial T}{\partial r}\right)+\frac{16 \sigma T_{\infty}^{3}}{3 \kappa} \frac{1}{\rho c_{p}} \frac{\partial}{\partial r}\left(r \frac{\partial T}{\partial r}\right) .
$$

Setting the following similarity transformations:

$$
\eta=\left(\frac{r}{x}\right)^{2} R a_{x}, \quad \psi=\alpha x f(\eta), \quad \theta=\frac{T-T_{\infty}}{T_{w}-T_{\infty}}, \quad \phi=\frac{C-C_{\infty}}{C_{w}-C_{\infty}},
$$

where $R a_{x}=\frac{x}{\alpha}\left[\frac{\rho_{\infty} g \beta_{0} K^{*}\left(T_{w}-T_{\infty}\right)}{\mu^{*}}\right]^{1 / n}$.

Employing (11) in (2), (4) and (10), we can derive the following coupled system of differential equations:

$$
\begin{gathered}
\left(n f^{\prime n-1}+2^{3-n} G r^{*} f^{\prime}\right) f^{\prime \prime}=\frac{1}{2^{n}}\left[\left(1+2 \alpha_{1} \theta\right) \theta^{\prime}+N\left(1+2 \alpha_{2} \phi\right) \phi^{\prime}\right] \\
\left(1+\frac{4}{3} R\right)\left(2 \eta \theta^{\prime \prime}+2 \theta^{\prime}\right)+f \theta^{\prime}=0 \\
\frac{1}{L e}\left(2 \eta \phi^{\prime \prime}+2 \phi^{\prime}\right)+f \phi^{\prime}=0
\end{gathered}
$$

along with the associated conditions

$$
\begin{array}{lll}
\text { at } & \eta=\eta_{0}: & f-\eta f^{\prime}=0, \theta=1, \phi=1, \\
\text { as } & \eta \rightarrow \infty: & f^{\prime}=0, \quad \theta=0, \phi=0 .
\end{array}
$$

In the above equations, $G r^{*}=\left(\frac{\rho_{\infty} b K^{*}}{\mu}\right)\left(\frac{\alpha}{x} R a_{x}\right)^{2-n}$ is the modified Grashof number, $R=\frac{4 \sigma^{*} T_{\infty}}{K^{*} \kappa}$ is the radiation parameter, $N=\frac{\beta_{2}\left(C_{w}-C_{\infty}\right)}{\beta_{0}\left(T_{w}-T_{\infty}\right)}$ is the buoyancy ratio, $\alpha_{1}=\frac{\beta_{1}\left(T_{w}-T_{\infty}\right)}{\beta_{0}}$ and $\alpha_{3}=\frac{\beta_{3}\left(C_{w}-C_{\infty}\right)}{\beta_{2}}$ are the nonlinear densitytemperature and nonlinear density-concentration parameters,respectively and $L e=$ $\frac{\alpha}{D}$ is the Lewis number (diffusivity ratio).

The Nusselt number and Sherwood number are defined, respectively, as $N u_{x}=$ $h x /\left(k\left(T_{w}-T_{\infty}\right)\right)$ and $S h_{x}=q x /\left(D\left(C_{w}-C_{\infty}\right)\right)$. Also the non-dimensional form of the Nusselt and Sherwood numbers may be written as

$$
\begin{gathered}
N u_{x} / R a_{x}^{1 / 2}=-2 \eta_{0}^{1 / 2} \theta^{\prime}\left(\eta_{0}\right)\left[1+\frac{4}{3} R\right], \\
S h_{x} / R a_{x}^{1 / 2}=-2 \eta_{0}^{1 / 2} \phi^{\prime}\left(\eta_{0}\right) .
\end{gathered}
$$

where $h\left(T_{w}-T_{\infty}\right)=\left[\left(16 \sigma^{*} T_{\infty}^{3} / 3 \kappa+k\right) \frac{\partial T}{\partial r}\right]$. 


\section{ANALYSIS OF RESULTS}

The system of coupled nonlinear differential equations (12) - (14) along with the boundary conditions (15) - (16) are solved numerically by using 4th order RungeKutta method together with the shooting technique. To validate the numerical computations, the values of non-dimensional heat transfer rate are compared (Fig. 2) with the previously published work of Lai et al. [9] in the case of linear Boussinesq approximation without considering the radiation effect in the Darcy porous media. In this investigation, the value $\eta_{\infty}=30$ is chosen in such a manner that the values of $f, \theta$ and $\phi$ at outer edge matches with the prescribed boundary conditions. The main intention of this analysis is to explore the combined effects of important flow influencing parameters, namely nonlinear temperature and nonlinear concentration parameters, inertia and radiation on the flow field, concentration and temperature distributions. Also, to figure out the associated changes in the mass and heat transfer rates due to the correlation of the major parameters. Figures are plotted for few selected values of the parameters and results are analyzed graphically.

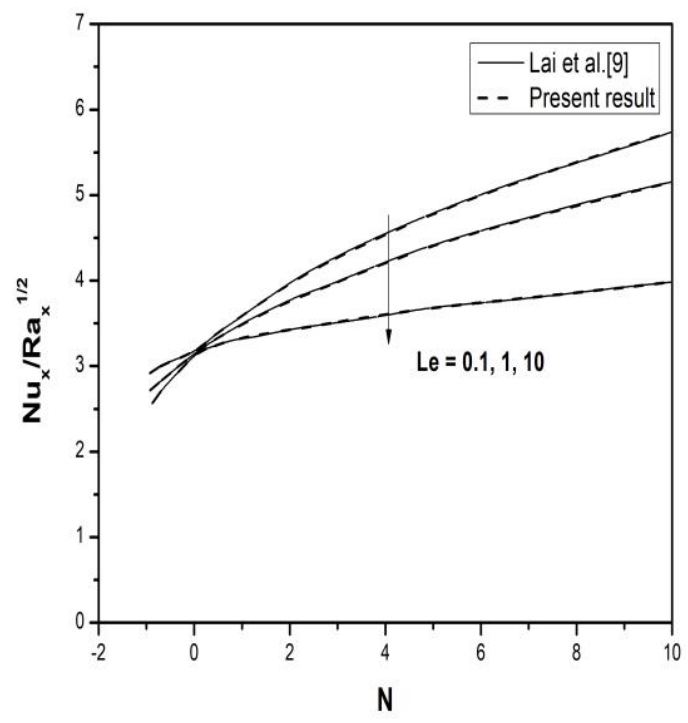

Fig. 2: Comparison of heat transfer coefficient for Newtonian fluid $(n=1)$ in a Darcy $\left(G r^{*}=0\right)$ porous medium in absence of radiation $(R=0)$.

The significance of nonlinear thermal and concentration parameters on dimensionless velocity, concentration and temperature distribution, is illustrated in Fig. (3), Fig. (4), and Fig. (5), respectively. It is observed that hydrodynamic boundary layer thickness increases with the increased values of power-law index $n$, nonlinear tem- 


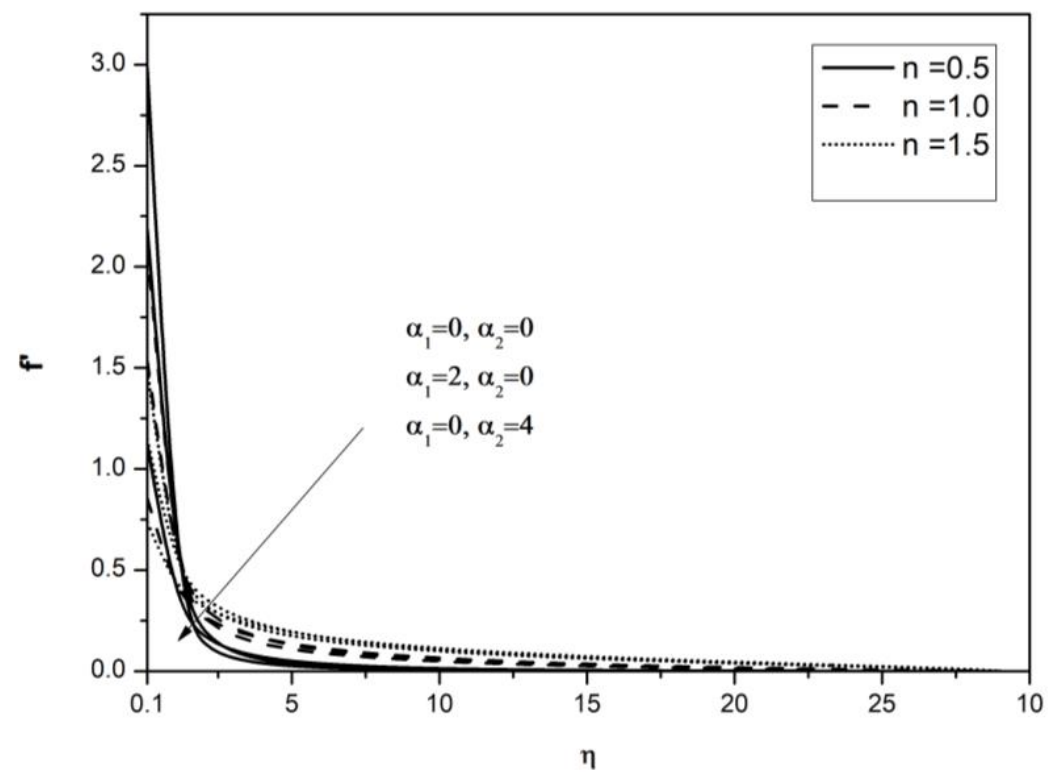

Fig. 3: Velocity profile against $\eta$ with with $G r^{*}=0.1, L e=1, R=0$ and $N=1$.

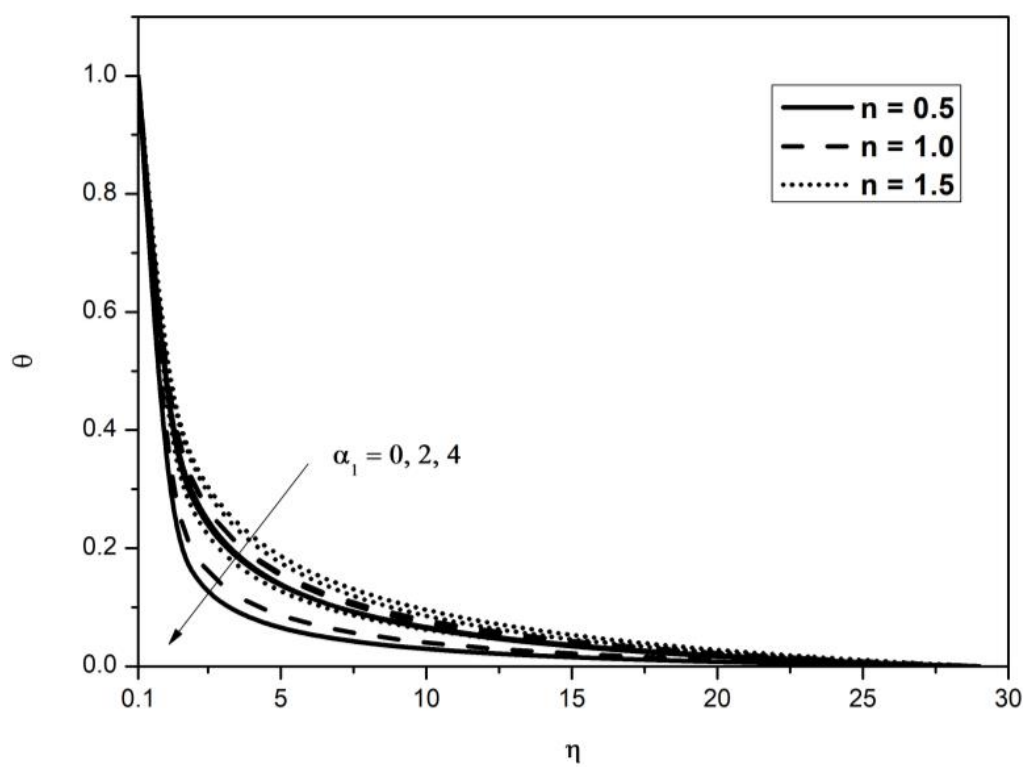

Fig. 4: Temperature profile against $\eta$ with $G r^{*}=0.1, L e=1, R=0$ and $N=1$. 


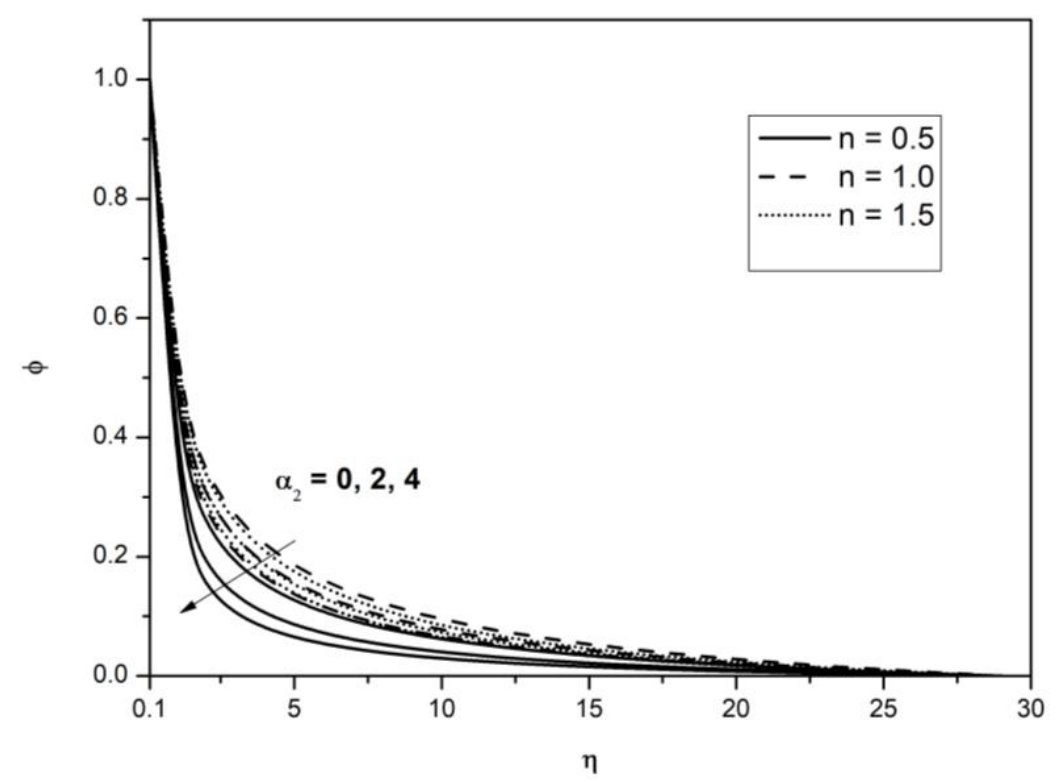

Fig. 5: Concentration profile against $\eta$ with $G r^{*}=0.1, L e=1, R=0$ and $N=1$.

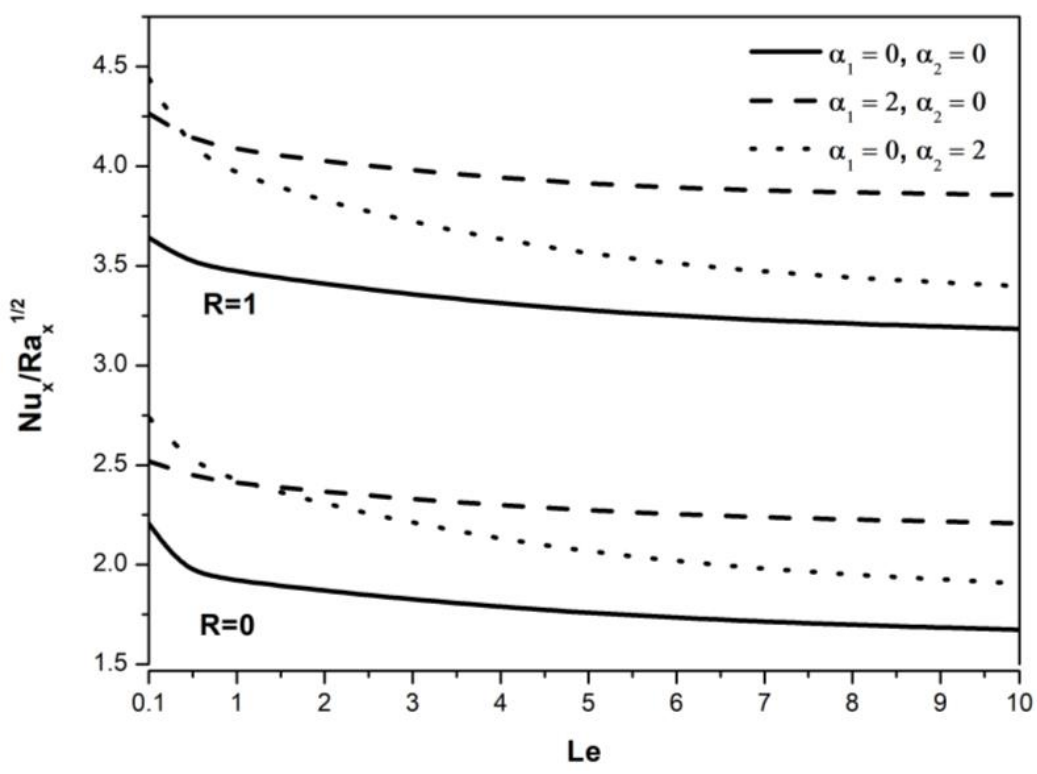

Fig. 6: The heat transfer coefficient as a function of $L e$ for $n=0.5$ with $G r^{*}=0.1$ and $N=1$. 
214 Nonlinear Double Diffusive Convection from a Radiative Slender ...

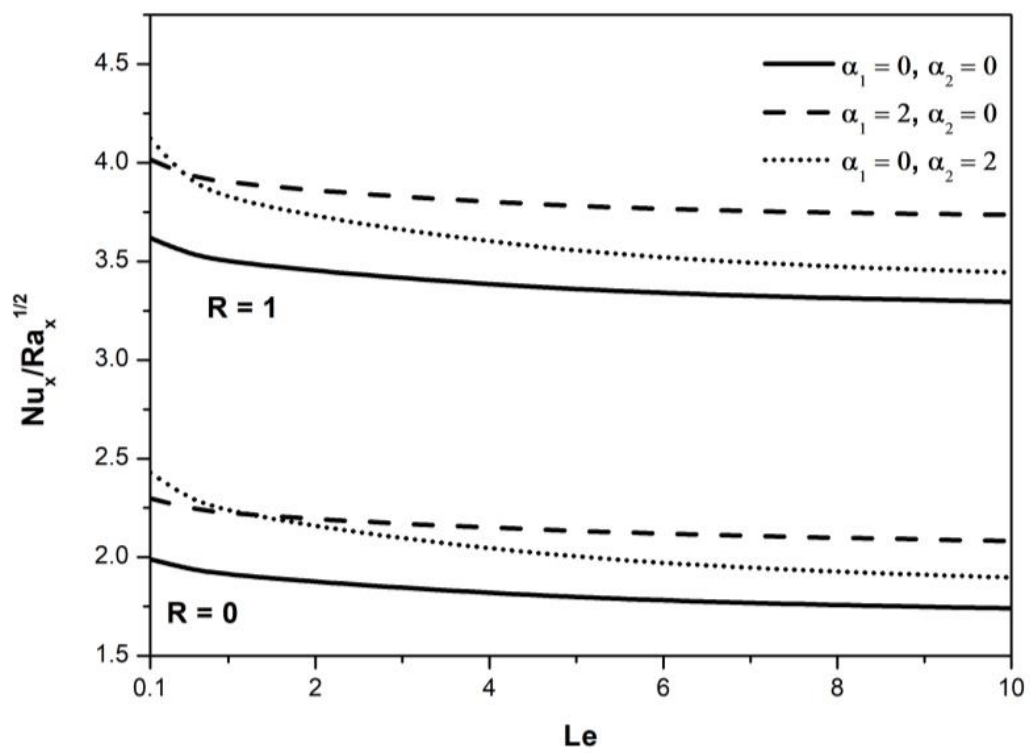

Fig. 7: The heat transfer coefficient as a function of $L e$ for $n=1.0$ with $G r^{*}=0.1$ and $N=1$.

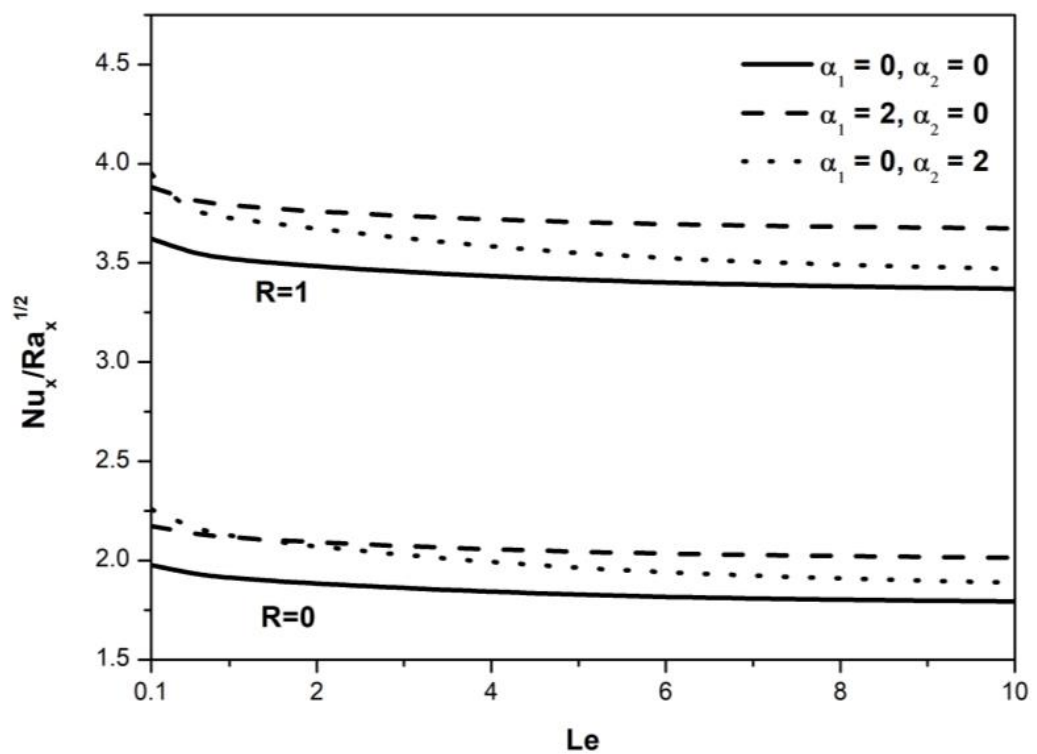

Fig. 8: The heat transfer coefficient as a function of $L e$ for $n=1.5$ with $G r^{*}=0.1$ and $N=1$. 
perature parameter $\alpha_{1}$ and nonlinear concentration parameter $\alpha_{2}$. The reason for such happening is as power-law index varies from $n<1$ to $n>1$, power-law fluid gradually becomes shear-thinning to shear-thickening which results increase in boundary layer thickness and hence flow velocity will be diminished. Moreover, for positive values of $\alpha_{1}, \alpha_{2}$ added nonlinearity into the momentum equation (2) and hence additional thermal and solutal density gradient are enhanced which also result the increase in boundary layer thickness too. It is also noticed in Figs. (4) and (5) that temperature and concentration boundary layer thicknesses are increased for increasing values of power-law index $n$. As a result, both heat and mass transfer rates decrease with increased values of $n$ (this will be displayed later in Figs. (11) and (12)). On the other hand, temperature and concentration boundary layer thicknesses are diminished with enhanced values of $\alpha_{1}$ and $\alpha_{2}$, respectively. Thus, Nusselt and Sherwood numbers are increased with $\alpha_{1}$ and $\alpha_{2}$ (this will be displayed later in Figs. (6) - (10)).

Figures (6) - (8) illustrate the variation of Nusselt number against the Lewis number for varying values of the radiation, nonlinear thermal and concentration parameters with fixed inertia and buoyancy parameter. It is noted that the non-dimensional heat transfer rate increases with increased value $R, \alpha_{1}$ and $\alpha_{2}$ for all possible values of $n$. Physically, the presence of radiation, supplies additional heat to the flow which leads the velocity and temperature to go up within the boundary layer region

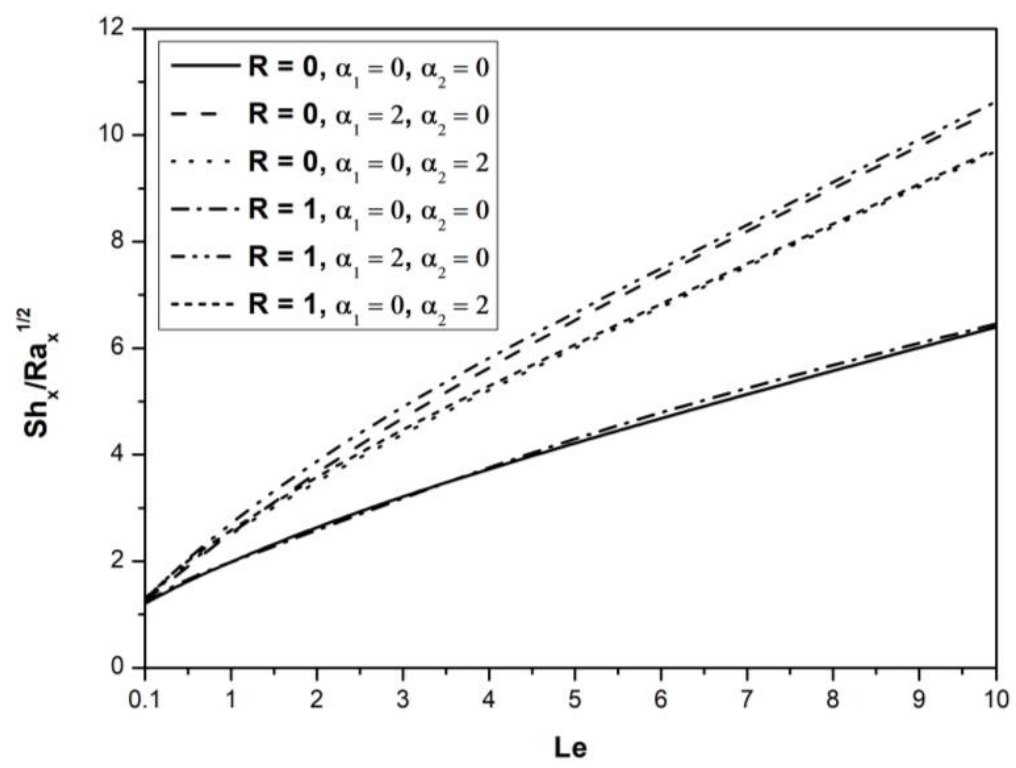

Fig. 9: The mass transfer coefficient as a function of $L e$ for $n=0.5$ with $G r^{*}=0.1$ and $N=1$. 


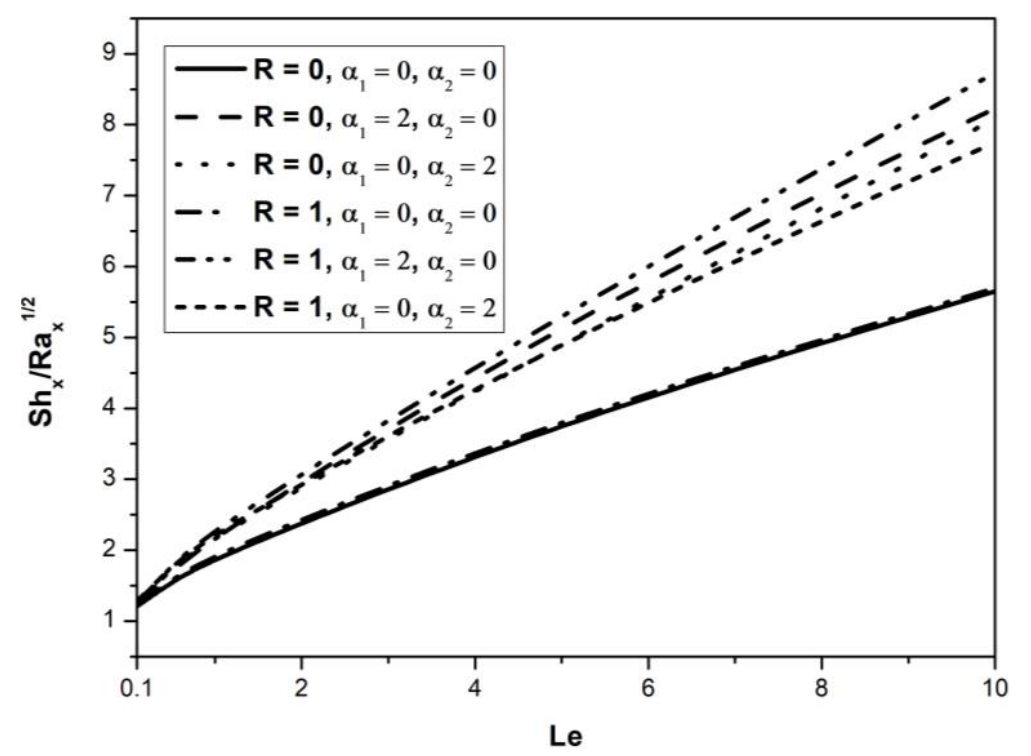

Fig. 10: The mass transfer coefficient as a function of $L e$ for $n=1.0$ with $G r^{*}=0.1$ and $N=1$.

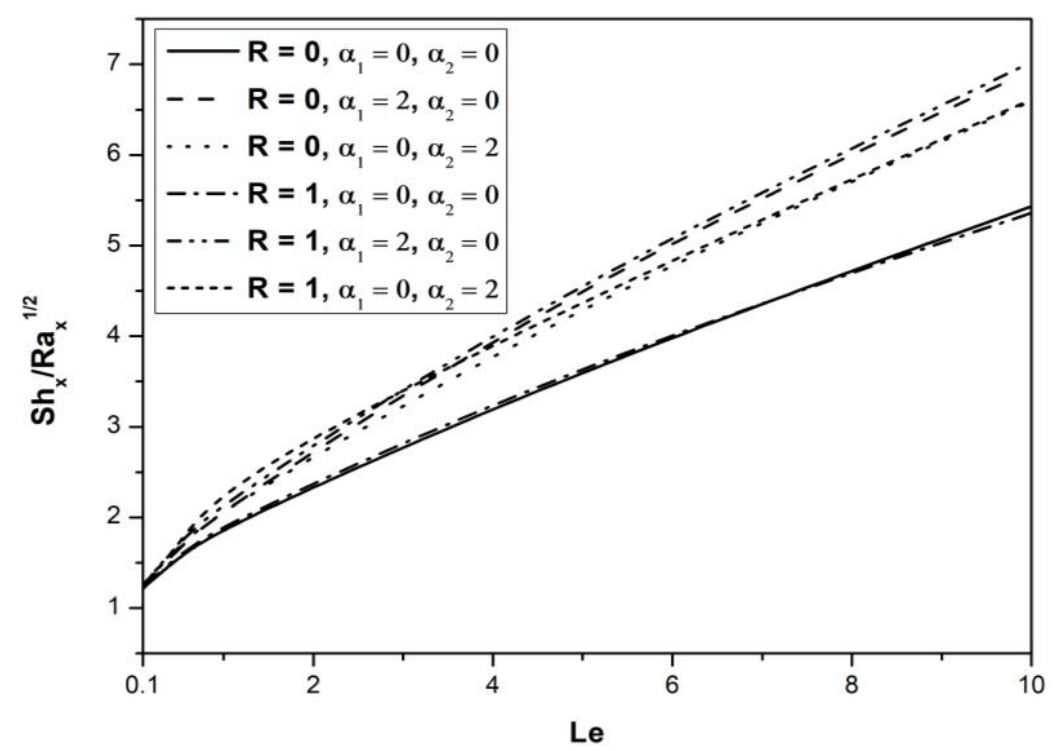

Fig. 11: The mass transfer coefficient as a function of $L e$ for $n=1.5$ with $G r^{*}=0.1$ and $N=1$. 


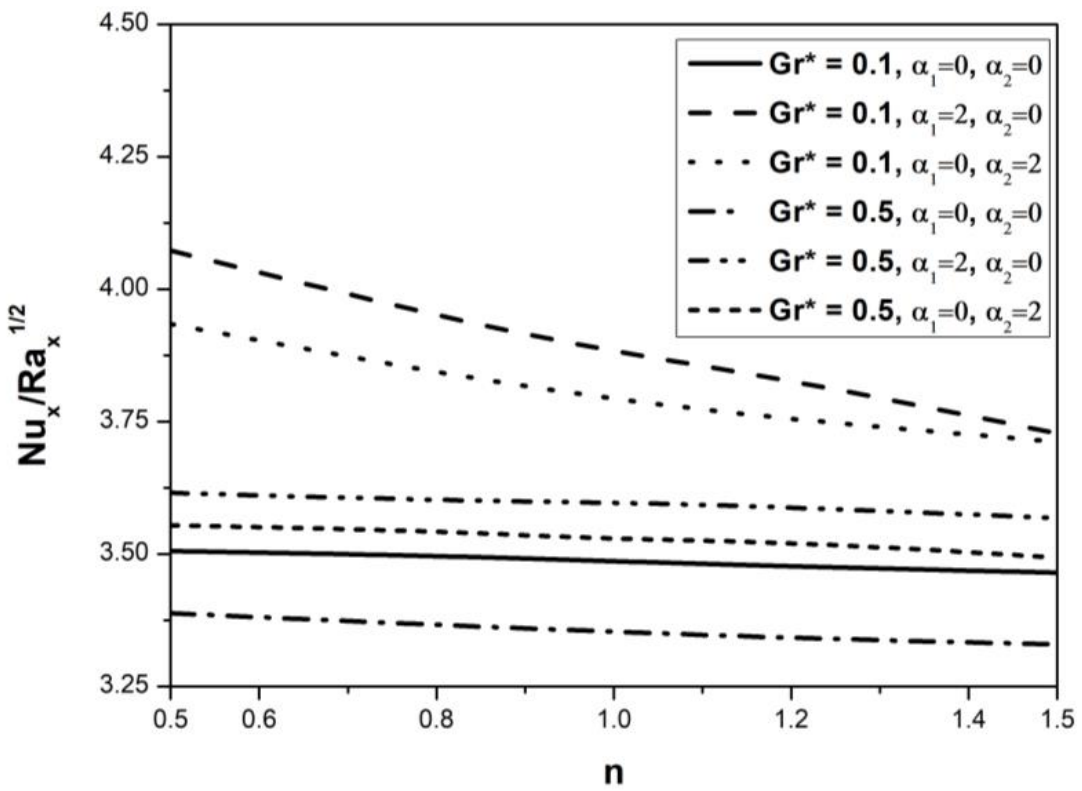

Fig. 12: The heat transfer coefficient as a function of $n$ with $R=1, L e=1$ and $N=1$.

consequently favours heat transfer and also gives explanation of significance of $\alpha_{1}$ and $\alpha_{2}$ on heat transfer as already described in Figs. (4) and (5). Also, the effectiveness of both nonlinear thermal and concentration parameters is significant in a porous medium in the presence of radiation. Moreover, the nonlinear parameters are prominent in the case of pseudoplastics fluids when compared to Newtonian and dilatant fluids saturated porous medium. Further, it is observed that the nonlinear concentration parameter plays a vital role for smaller $L e$ in the case of both Newtonian and non-Newtonian fluids.

In Fig. (9), Fig. (10), and Fig. (11) the Sherwood number is plotted as a function of $L e$ for $n=0.5, n=1.0$ and $n=1.5$, respectively varying $R, \alpha_{1}$ and $\alpha_{2}$. From these figures, it is seen that the mass transfer rate increases with increased values of $L e, \alpha_{1}$ and $\alpha_{2}$. As in the case of heat transfer rate, the nonlinear temperature and concentration parameters are prominent for pseudoplastic fluid when compared to Newtonian and dilatant fluids. Also, the role of nonlinear parameters is amplified with larger values of $L e$. Furthermore, the upshot due to nonlinear convection parameters on mass transfer rate is more for a pseudoplastic fluid when compared to Newtonian and dilatant fluids saturated porous medium. On the contrary, the radiation plays a very obvious role on mass transfer rate. 


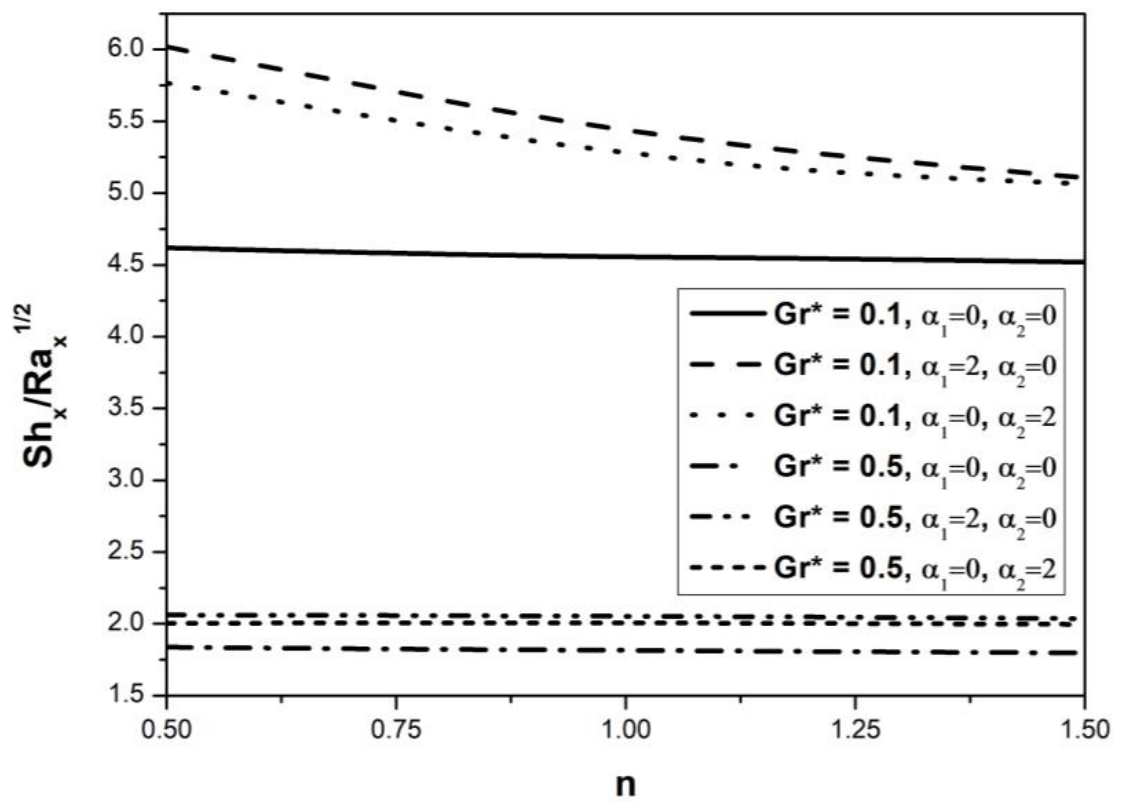

Fig. 13: The mass transfer coefficient as a function of $n$ with $R=1, L e=1$ and $N=1$.

Figures (12) and (13), respectively, depict the variation of Nusselt and Sherwood numbers against $n$ for different values of $G r^{*}, \alpha_{1}$ and $\alpha_{2}$. It is observed the the inertia parameter $G r^{*}$ decelerates both the heat and mass transfer rates. The reason for such happening is due to rise in hydrodynamic boundary layer thickness which results a reduction in velocity inside the boundary layer for all possible values of $n$. The significance of nonlinear parameters on the Nusselt and Sherwood numbers is less effective for higher inertial porous medium. Furthermore, for larger values of $G r^{*}$, the non-dimensional Nusselt and Sherwood numbers vary almost linearly with $n$.

\section{CONCLUSIONS}

Nonlinear double diffusion convection on account of radiation near a slender paraboloid in a non-Darcy porous media saturated by a Ostwald-de Waele power-law fluid, is analyzed in this work. The resultant governing nonlinear coupled differential equations are solved utilizing the shooting technique along with the 4th order Runge-Kutta method. The role of major flow controlling parameters on flow, concentration, temperature, and as well as on the mass and heat transfer rates, are illustrated graphically. It is found that the non-dimensional Sherwood and Nusselt numbers 
are enhanced with the nonlinear temperature, concentration and radiation parameters while those decrease with the power-law index. One of the major observations is that the Lewis number plays a vital role on the heat and mass transfer rates in the presence of nonlinear convection. Finally, the nonlinear concentration and temperature parameters on mass and heat transfer rates are less effective for higher value of inertia parameter.

\section{ACKNOWLEDGEMENTS}

Dr. Rishi Raj Kairi is thankful to the UGC, India, for financial support in pursuing this research work.

\section{REFERENCES}

[1] H.T. ChEN, C.K. CHEN (1988) Natural Convection of a Non-Newtonian Fluid About a Horizontal Cylinder and Sphere in a Porous Medium. International Communications in Heat and Mass Transfer 15 605-614.

[2] H.T. Chen, C.K. Chen (1988) Natural Convection of Non-Newtonian Fluids along a Vertical Plate Embedded in a Porous Medium. ASME Journal of Heat Transfer 110 257-260.

[3] H.K. Mohanty (1978) Similarity Solutions of Unsteady Laminar, Incompressible Boundary Layer Equations for Flow, Heat and Mass Transfer in Non-Newtonian Fluids around Axisymmetric Bodies. Rheological Acta 17(4) 342-352.

[4] M.J. BabU, N. SAndeEP (2016) MHD Non-Newtonian Fluid Flow over a Slendering Stretching Sheet in the presence of Cross-Diffusion Effects. Alexandria Engineering Journal 55(3) 2193-2201.

[5] A. Nakayama, H. Koyama (1991) Buoyancy Induced Flow of Non-Newtonian Fluids over a Non-Isothermal Body of Arbitrary Shape in a Fluid-Saturated Porous Medium. Applied Scientific Research 48 55-70.

[6] A.V. Shenoy (1993) Darcy-Forchheimer Natural, Forced and Mixed Convection Heat Transfer in Non-Newtonian Power-Law Fluid-Saturated Porous Media. Transport in Porous Media 11 219-241.

[7] A.A. Mohammadein, M.F. El-Amin (2000) Thermal Radiation Effect on Power Law Fluid over a Horizontal Plate Embedded in a Porous Medium. International Communications in Heat and Mass Transfer 27 1025-1035.

[8] A. Nakayama, H. Koyama (1987) Free Convective Heat Transfer over a NonIsothermal Body of Arbitrary Shape Embedded in a Fluid Saturated Porous Medium. ASME Journal of Heat Transfer 109 125-130.

[9] F.C. Lai, C.Y. ChOI, F.A. Kulacki (1990) Coupled Heat and Mass Transfer by Natural Convection from a Slender Bodies of Revolution in Porous Media. International Communications in Heat and Mass Transfer 17 609-620. 
[10] B.B. Singh, I.M. Chandarki (2009) Integral Treatment of Coupled Heat and Mass Transfer by Natural Convection from a Cylinder in Porous Media. International Communications in Heat and Mass Transfer 36 269-273.

[11] A.V. Shenoy (1994) Non-Newtonian Fluid Heat Transfer in Porous Mediua. Advances in Heat Transfer 24 102-184.

[12] J.R. Reddy, V. Sugunamma, N. Sandeep (2017) Enhanced Heat Transfer in the Flow of Dissipative Non-Newtonian Casson Fluid Flow over a Convectively Heated Upper Surface of a Paraboloid Revolution. Journal of Molecular Liquids 229 380-388.

[13] J.R. Reddy, V. Sugunamma, N. Sandeep (2017) Effect of Frictional Heating on Radiative Ferro Fluid Flow over a Slendering Stretching Sheet with Aligned Magnetic Field. The European Physical Journal Plus 132(1) 7.

[14] A. RAptis (1998) Radiation and Free Convecton Flow through a Porous Medium. Intern. Communications in Heat and Mass Transfer 25 289-295.

[15] D. Srinivasacharya, Ch. Ramreddy, P. Naveen (2019) Effect of Nonlinear Boussinesq Approximation and Double Dispersion on a Micropolar Fluid Flow under Convective Thermal Condition. Heat transfer Asian Research 48(1) 414-434.

[16] Ch. Ramreddy, P. NAVEen, D. Srinivas ACharya (2019) Influence of Nonlinear Boussinesq Approximation on Natural Convective Flow of a Power-Law Fluid along a Inclined Plate under Convective Thermal Boundary Condition. Nonlinear Engineering 8 94-106.

[17] M.K. Partha (2010) Nonlinear Convection in a Non-Darcy Porous Medium. Applied Mathematics and Mechanics 31 565-574.

[18] I. Mandal, S. Mukhopadhyay (2019) Nonlinear Convection in Micropolar Fluid Flow Past an Exponential Moving Stream with Thermal Radiation. Mechanics of Advanced Materials and Structures 26 2040-2046.

[19] M. Modather, A.M. Rashad, A.J. Chamkha (2009) An Analytical Study on MHD Heat and Mass Transfer Oscillatory Flow of Micropolar Fluid over a Vertical Permeable Plate in a Porous Medium. Turkish Journal of Engineering and Environmental Sciences 33 245-257.

[20] A.M. Rashad, A.J. Chamkha, S.M.M. El-Kabier (2011) Effect of Chemical Reaction on Heat and Mass Transfer by Mixed Convection Flow about a Solid Sphere in a Saturated Porous Media. International Journal of Numerical Methods for Heat and Fluid Flow 21(4) 418-433.

[21] H.A. NABWAY, S.M.M. El-KAbEIR, A.M. Rashad (2015) Lie Group Analysis of Effects of Radiation and Chemical Reaction on Heat and Mass Transfer by Unsteady Slip Flow From a Non-Isothermal Stretching Sheet Immersed in a Porous Medium. Journal of Computational and Theoretical Nanoscience 12(11) 4056-4062.

[22] B. Mallikarjuna, A.M. Rashad, A.J. Chamkha, S.H. Raju (2016) Chemical Reaction Effects On MHD Convective Heat and Mass Transfer Flow Past a Rotating Vertical Cone Embedded in a Variable Porosity Regime. Afrika Matematika 27(3) 646665 . 
[23] R.R. KAIRI (2019) Free Convection around a Slender Paraboloid of Non-Newtonian Fluid in a Porous Medium. Thermal Science 23 3067-3074.

[24] R.H. Christopher, S. Middleman (1965) Power Law Fluid Flow Through a Packed Tube. Industrial \& Engineering Chemistry Fundamentals 4 422-426.

[25] R.V. Dharmadhikari, D.D. Kale (1985) The Flow of Non-Newtonian Fluids through Porous Media. Chemical Engineering Science 40 527-529. 